\title{
Efficiency of Brownian Motors
}

\author{
J.M.R. Parrondo \\ Dept. de Física Atómica, Nuclear y Molecular, Universidad Complutense, 28040-Madrid, Spain \\ J.M. Blanco, F.J. Cao, and R. Brito \\ Dept. de Física Aplicada I, Universidad Complutense, 28040-Madrid, Spain
}

February 1, 2008

Pacs: $05.40,82.20 \mathrm{M}$

\begin{abstract}
The efficiency of different types of Brownian motors is calculated analytically and numerically. We find that motors based on flashing ratchets present a low efficiency and an unavoidable entropy production. On the other hand, a certain class of motors based on adiabatically changing potentials, named reversible ratchets, exhibit a higher efficiency and the entropy production can be arbitrarily reduced.
\end{abstract}

In the last years there has been an increasing interest in the so-called "ratchets" or Brownian motors 11, 2, 3, 4, 5, 6. These systems consist of Brownian particles moving in asymmetric potentials, such as the one depicted in fig. 1 (left), and subject to a source of non-equilibrium, like external fluctuations or temperature gradients. As a consequence of these two ingredients - asymmetric potentials and non-equilibrium-, a flow of particles can be induced.

Most of the cited papers consider systems where the Brownian particles do not gain energy in a systematic way. Although these systems are called "Brownian or molecular motors", they do not convert heat into work, nor induce any energy conversion. Feynman in his Lectures [7] already understood that, in order to have an engine out of a ratchet, it is necessary to use its systematic motion to store potential energy. This can be achieved if the ratchet lifts a load. Then the ratchet becomes a thermal engine and Feynman estimated its efficiency (although following assumptions which have been revealed to contain some inconsistencies [8]). Recently, Sekimoto [9] has generalized this procedure, defining efficiency for a wide class of ratchets. Jülicher et al [6] have also discussed the efficiency of molecular motors and Sokolov and Blumen 10 have calculated the efficiency of a deterministically flashing ratchet in contact with thermal baths at different temperatures. A general conclusion is that these motors are intrinsically irreversible, even in the quasistatic limit [6, 8, 9, 10].

On the other hand, it has been recently introduced [11] a class of deterministically driven ratchets where the entropy production vanishes in the quasistatic limit, i.e., reversible ratchets. The aim of this letter is to explore the differences, regarding efficiency, between randomly flashing ratchets and both reversible and irreversible deterministically driven ratchets.

\section{Randomly flashing ratchets}

Consider two species of Brownian particles, say $A$ and $B$, moving in the interval $[0, L]$ with periodic boundary conditions. Particles of type $A$ feel a potential $V_{A}(x)$, whereas particles $B$ feel $V_{B}(x)$. Besides, there is a continuous transfer of particles, $A \rightleftharpoons B$, which accounts for non-equilibrium fluctuations. This picture is equivalent to that of a single Brownian particle in a randomly switching potential [3]. 

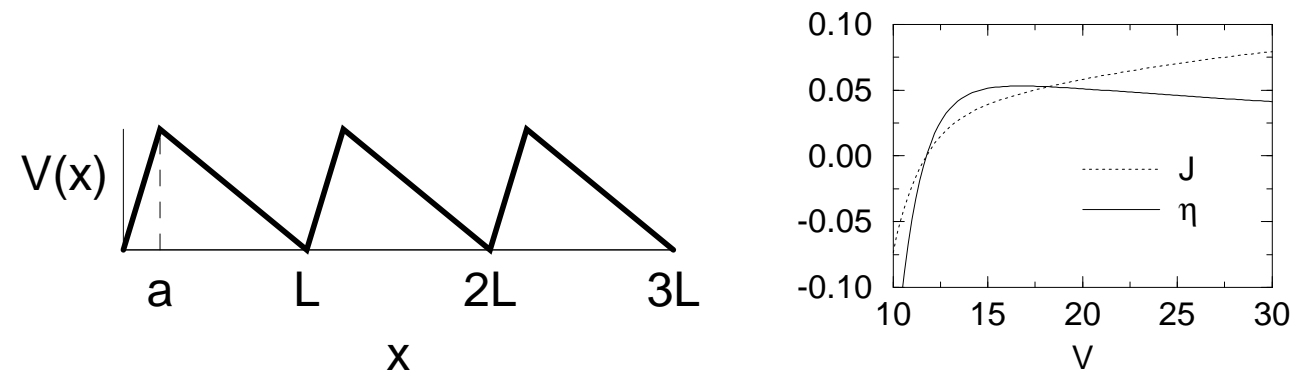

Figure 1: Left: Asymmetric sawtooth potential of the ratchets presented in refs. [1, 2, 3, 因, 5]. In this letter we consider two types of ratchets: a) one where the potential is randomly switched on and off; and b) one where the potential is deterministically modulated.

Right: Efficiency and current of the ratchet where the the potential on the left is randomly switched on and off (case a), as a function of the maximum height $V$ of the potential. The reaction rates are $\omega_{A}=1.08$ and $\omega_{B}=81.8, a=1 / 11$, and the external force is $F=4.145$.

In ref. [3], it was proved that a flow towards a given direction, say, to the right, occurs for some asymmetric potentials $V_{A}$ and $V_{B}$. If we add a load or force $F$ opposite to the flow, the evolution equation for the probability density of particles $A, \rho_{A}(x)$, and particles $B, \rho_{B}(x)$, is:

$$
\begin{aligned}
& \partial_{t} \rho_{A}(x, t)=-\partial_{x} \mathcal{J}_{A} \rho_{A}(x, t)-\omega\left[\rho_{A}(x, t)-\rho_{B}(x, t)\right] \\
& \partial_{t} \rho_{B}(x, t)=-\partial_{x} \mathcal{J}_{B} \rho_{B}(x, t)+\omega\left[\rho_{A}(x, t)-\rho_{B}(x, t)\right]
\end{aligned}
$$

where $\mathcal{J}_{i}=-V_{i}^{\prime}(x)-F-\partial_{x}$ is the current operator, the prime indicates derivative with respect to $x$, and $\omega$ is the rate of the reaction $A \rightleftharpoons B$. We have taken units of energy, length and time such that the temperature is $k_{B} T=1$, the length of the interval is $L=1$, and the diffusion coefficient is $D=1$.

The flow of particles in the stationary regime is $J=\mathcal{J}_{A} \rho_{A}^{s t}(x)+\mathcal{J}_{B} \rho_{B}^{s t}(x)$, where $\rho_{A, B}^{s t}(x)$ are the stationary solutions of eq. (11). This flow $J$ is a decreasing function of the external force $F$ and becomes negative if $F$ is stronger than a balancing force, $F_{b a l}$. Therefore, if $0<F<F_{b a l}$, particles move against the force and, consequently, gain potential energy in a systematic way. The potential energy gain or output energy per unit of time is $E_{\text {out }}=J F$, which vanishes both for $F=0$ and $F=F_{b a l}$.

On the other side, switching on and off the potential requires some energy. In our two-species picture, the reaction $A \rightleftharpoons B$ does not conserve energy since $V_{A}(x) \neq V_{B}(x)$. Therefore, in each reaction $A \rightarrow B$, occurring at a point $x$, an energy $V_{B}(x)-V_{A}(x)$ is transferred to the system (or withdrawn, if the sign is negative). Similarly, an energy $V_{A}(x)-V_{B}(x)$ is transferred to the system in each reaction $B \rightarrow A$ occurring at $x$. In the stationary regime, the average number of such reactions per unit of time is, respectively, $\omega \rho_{A}^{s t}(x)$ and $\omega \rho_{B}^{s t}(x)$. Therefore, the input energy per unit of time is 6 , 9]:

$$
E_{\text {in }}=\omega \int_{0}^{1} d x\left[V_{B}(x)-V_{A}(x)\right]\left[\rho_{A}^{s t}(x)-\rho_{B}^{s t}(x)\right] .
$$

Finally, the efficiency can be defined as [6, 9]:

$$
\eta=\frac{E_{\text {out }}}{E_{\text {in }}} .
$$

This efficiency can be calculated analytically for the system given by eq. (11) with piecewise potentials. We have performed an exhaustive study for the particular setting $V_{B}(x)=0$ and $V_{A}(x)$ 
equal to the potential depicted in fig. 1 1 (left):

$$
V_{A}(x)= \begin{cases}V x / a & \text { if } x \leq a \\ V(1-x) /(1-a) & \text { if } x \geq a\end{cases}
$$

with $a=1 / 11$. For this system, the maximum efficiency is $\eta_{\max }=3.29 \%$, which is reached for $V=22, F=3$, and $\omega=63$. This efficiency can be improved using different reaction rates: $\omega_{A}$ for $A \rightarrow B$ and $\omega_{B}$ for $B \rightarrow A$. In this case, $\eta_{\max }=5.315 \%$ with $V=16.7, F=4.145, \omega_{A}=1.08$, and $\omega_{B}=81.8$. Observe that, with these values for $\omega_{A, B}$, the particle stays much longer within the potential $V_{A}(x)$ than within $V_{B}(x)$.

We have plotted in fig. 1 (right) the efficiency and the flow of particles as a function of $V$ setting the rest of parameters equal to these optimal values. Two are the messages from this figure. Firstly, the maximization of the efficiency is a new criterion to define optimal Brownian motors and this criterion is, in some cases, less trivial than that of maximizing the flow. Secondly, the randomly flashing ratchet under study has a rather low efficiency. As we have mentioned before, the heat dissipation per unit of time is $E_{\text {in }}-E_{\text {out }}$. Consequently, the increase of entropy of the thermal bath, per unit of time, is $E_{\text {in }}-E_{\text {out }}$, since $k_{B} T=1$. On the other hand, in the stationary regime there is no change of entropy in the system nor in the external agent which provides the non-equilibrium fluctuations 1. Therefore, the net entropy production per unit of time is $E_{\text {in }}-E_{\text {out }}$. If this entropy production would vanish, i.e., if the system could work in a reversible way, it should reach a $100 \%$ efficiency. On the contrary, the efficiency is below $10 \%$ and we can conclude that the motor based on the randomly flashing ratchet is very inefficient.

One could think that the efficiency would increase in limiting situations where the system is close to equilibrium, such us $\omega \rightarrow 0$ and/or $V_{A}-V_{B} \rightarrow 0$. However, a perturbative analysis of eq. (11) shows that $\eta \rightarrow 0$ in both limits. In the first case, $\omega \rightarrow 0$, from eq. (11) one can easily find that $J$ is of order $\omega$, so is $F_{b a l}$. Therefore, $E_{\text {out }}$, in the interval $0<F<F_{b a l}$, is of order $\omega^{2}$, whereas one can prove that $E_{i n}$ is of order $\omega$, giving a zero efficiency in this limit. In the second case, $\Delta V(x) \equiv V_{A}(x)-V_{B}(x) \rightarrow 0$, the input energy $E_{i n}$ is of order $\Delta V^{2}$. However, surprisingly enough, $J$ is of order $\Delta V^{2}$ and so is $F_{b a l}$, yielding $E_{\text {out }}$ of order $\Delta V^{4}$ and, again, a vanishing efficiency. We conclude that the flashing motor is intrinsically irreversible, as it has been pointed out for related models in refs. [6, 8, 9, 10].

\section{Deterministically driven ratchets}

A different strategy to reduce the production of entropy consists of considering Brownian particles in a potential which changes deterministically in time. If the potential is changed very slowly, the system evolves close to equilibrium and the entropy production is low. From now on, we focus our attention on Brownian particles in a spatially periodic potential $V(x ; \mathbf{R}(t))$ depending on a set of parameters collected in a vector $\mathbf{R}$ which changes in time [11]. The parameters are changed periodically in time with period $T$, i.e., $\mathbf{R}(0)=\mathbf{R}(T)$.

As in ref. [9], we have to modify our definition of efficiency. Firstly, we deal with energy transfer per cycle $[0, T]$ instead per unit of time. Secondly, the input energy or work done to the system in a cycle, as a consequence of the change of the parameters $\mathbf{R}(t)$, is:

$$
E_{\text {in }}=\int_{0}^{T} d t \frac{\partial V(x ; \mathbf{R}(t))}{\partial t} \rho(x, t) .
$$

The probability density $\rho(x, t)$ verifies the Smoluchowski equation:

$$
\partial_{t} \rho(x, t)=-\partial_{x} \mathcal{J}_{\mathbf{R}(t)} \rho(x, t)
$$

\footnotetext{
${ }^{1}$ A physical realization of this external agent is a third species of particles, say $C$, feeling a potential $V_{C}(x)=$ $V_{B}(x)-V_{A}(x)$ and participating in the reaction as $A+C \rightleftharpoons B$. If the temperature of $C$ particles is the same as $B$ and $A$ particles, then detailed balance holds and there is no flow of particles. However, if the temperature of the $C$ particles is infinity, we recover the flashing ratchet discussed in the text. Therefore, this randomly flashing ratchet can be considered as a thermalengine in contact with two thermal baths, one at $T=1 / k_{B}$ and the other one at infinite temperature (see also 10 for an interpretation of the deterministically flashing ratchet as a thermal engine).
} 
where $\mathcal{J}_{\mathbf{R}}=-V^{\prime}(x ; \mathbf{R})-F-\partial_{x}$ is the current operator corresponding to the potential $V(x ; \mathbf{R})$. As before, the output energy is the current times the force $F$, but now the current is not stationary and we have to integrate along the process:

$$
E_{\text {out }}=\int_{0}^{T} d t F \mathcal{J}_{\mathbf{R}(t)} \rho(x, t)=F \phi .
$$

where $\phi$ is the integrated flow.

With the above expressions, the efficiency of the system, $\eta=E_{\text {out }} / E_{\text {in }}$, can be found analytically for $T$ large and weak external forces, where it is expected to be high. For the integrated flow one finds $\phi=\phi_{0}-\bar{\mu} F T$, where $\bar{\mu}$ is the average mobility of the system:

$$
\bar{\mu}=\frac{1}{T} \int_{0}^{T} \frac{d t}{Z_{+}(\mathbf{R}(t)) Z_{-}(\mathbf{R}(t))}
$$

and $\phi_{0}$ is the integrated flow for $F=0[1]$ :

$$
\phi_{0}=\oint d \mathbf{R} \cdot \int_{0}^{1} d x \int_{0}^{x} d x^{\prime} \rho_{+}(x ; \mathbf{R}) \nabla_{\mathbf{R}} \rho_{-}\left(x^{\prime} ; \mathbf{R}\right),
$$

with

$$
\rho_{ \pm}(x ; \mathbf{R})=\frac{e^{ \pm V(x ; \mathbf{R})}}{Z_{ \pm}(\mathbf{R})} ; \quad Z_{ \pm}(\mathbf{R})=\int_{0}^{1} d x e^{ \pm V(x ; \mathbf{R})} .
$$

In eq. (9) the contour integral runs over the closed path $\{\mathbf{R}(t): t \in[0, T]\}$ in the space of parameters of the potential. The term proportional to $T$ in the integrated flow, $\phi=\phi_{0}-\bar{\mu} F T$, arises because the force $F$ induces a non-zero current which is present along the whole process. As a consequence, the balancing force is $F_{b a l}=\phi_{0} / \bar{\mu} T$, and, in order to design a high efficiency motor, it is necessary to take simultaneously the adiabatic limit $T \rightarrow \infty$ and the limit $F \rightarrow 0$ with FT finite. Notice also that the above expressions are useless if $\phi_{0}=0$. In a previous paper [11], we have discussed the conditions for $\phi_{0}$ to be different from zero and called reversible ratchets those systems where $\phi_{0} \neq 0$. From now on, we restrict our analytical calculations to reversible ratchets, although we present below numerical results for an irreversible ratchet.

The input energy for weak force $F$ and large $T$ is $E_{i n}=\phi_{0} F+b / T$, with

$$
\begin{aligned}
b & =-\int_{0}^{T} d t Z_{-}(\mathbf{R}(t)) Z_{+}(\mathbf{R}(t))\left\{\left[\int_{0}^{1} d x \int_{0}^{x} d x^{\prime} \rho_{+}(x ; \mathbf{R}(t))\left[\partial_{t} \rho_{-}\left(x^{\prime} ; \mathbf{R}(t)\right)\right]\right]^{2}\right. \\
& \left.+\int_{0}^{1} d x \int_{0}^{x} d x^{\prime} \int_{0}^{x^{\prime}} d x^{\prime \prime}\left[\partial_{t} \rho_{-}(x ; \mathbf{R}(t))\right] \rho_{+}\left(x^{\prime} ; \mathbf{R}(t)\right)\left[\partial_{t} \rho_{-}\left(x^{\prime \prime} ; \mathbf{R}(t)\right)\right]\right\}
\end{aligned}
$$

which is a positive quantity. Combining the above expressions, one finds for the efficiency:

$$
\eta=\frac{F\left(\phi_{0}-\bar{\mu} F T\right)}{\phi_{0} F+b / T}=\frac{\phi_{0} \alpha-\bar{\mu} \alpha^{2}}{\phi_{0} \alpha+b}
$$

where $\alpha=F T$. This expression is exact in the limit $T \rightarrow \infty, F \rightarrow 0$. Notice that, even for large $T$, the irreversible contribution, $b / T$, to $E_{i n}$ is of the same order as $\phi_{0} F$.

In a given system, i.e., for a set of parameters $\phi_{0}, \bar{\mu}$ and $b$, the maximum efficiency is reached for $\alpha=\left(b / \phi_{0}\right)\left[\sqrt{1+\phi_{0}^{2} /(\bar{\mu} b)}-1\right]$ and its value is given by

$$
\eta_{\max }=1-2[\sqrt{z(1+z)}-z]
$$

with $z=b \bar{\mu} / \phi_{0}^{2}$. Eq. (12) clearly shows how the term $b$ in the denominator of eq. (11) prevents the system to reach an efficiency equal to one. Fortunately, as we will see below in a concrete example, using strong potentials one can get arbitrarily close to $100 \%$ efficiency. 

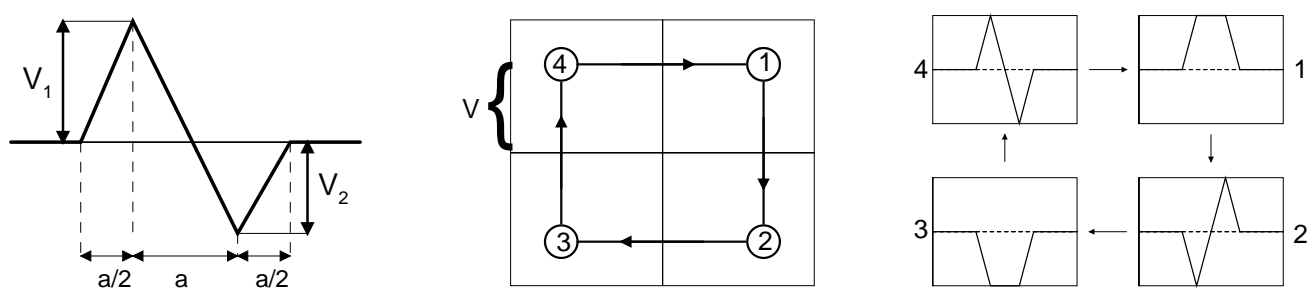

Figure 2: Graphical representation of the reversible ratchet described in the text: the potential depends on two parameters, $V_{1}$ and $V_{2}$, which are the height of two barriers/wells (left) and they change along the path depicted on the center ( $V$ being the maximum height/depth of the barriers/wells). On the right, the shape of the potential at the four labelled points.

To check the validity of the above theory and stress the differences between reversible and irreversible ratchets, we have studied in detail one example of each class.

As an example of irreversible ratchet, consider the modulation of the potential in fig. 1 (left), i.e., $V(x ; t)=\cos ^{2}(\pi t / T) V(x)$ with $V(x)$ given by eq. (1). In this case, $\phi_{0}$ is zero and the above theory cannot be applied. We have numerically integrated the Smoluchowski equation, eq. (6), using an implicit scheme with $\Delta t=10^{-5}, \Delta x=0.002,0.005$, and the Richardson extrapolation method to correct inaccuracies coming from the finite $\Delta x$. The efficiency has been obtained using eqs. (3), (5), (7) and the results, as a function of $F$ and for different values of $T$, are plotted in fig. 3 (left). The efficiency is maximum for $T$ around 0.5 and it goes to zero as $T$ increases. The maximum efficiency found by numerical integration is of the same order of magnitude as the one found for the randomly flashing ratchet. Notice, however, that we cannot explore with numerical experiments the whole space of parameters.
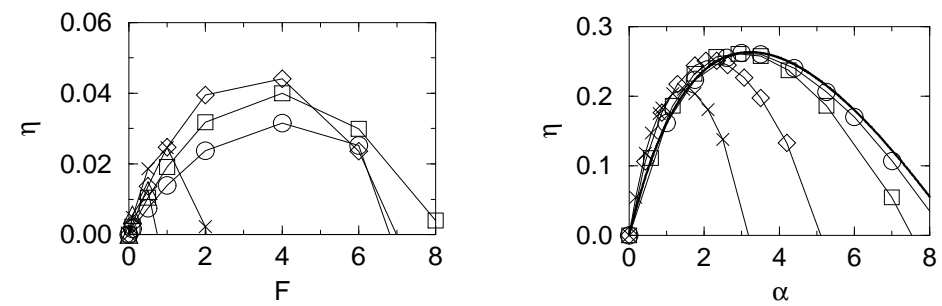

Figure 3: Left (irreversible ratchet): numerical results for the efficiency of the ratchet consisting of the potential in fig. 1 (left) modulated by $z(t)=\cos ^{2}(\pi t / T)$ as a function of the external force $F$ and for different values of the period $T: T=0.00125(\bigcirc), 0.025(\square), 0.05(\diamond), 0.25(\times)$, and 0.5 $(\triangle)$.

Right (reversible ratchet): numerical and analytical results for the efficiency of the ratchet described in fig. 2 for $V=5 a=0.2$ as a function of $F$ and for different values of the period $T: T=1(\times)$, $2(\diamond), 10(\square), 40(\bigcirc)$. The thick solid line is the analytical result given by eq. (11) in the limit $T \rightarrow \infty$ and $F \rightarrow 0$. Note that $\eta$ is an increasing function of $T$ in the reversible ratchet (right) as opposite to the irreversible case (left).

On the other hand, let us consider the reversible ratchet represented in fig. 2. Here the potential depends on two parameters, $V_{1}$ and $V_{2}$, which are the heights/depths of two triangular barriers/wells of width $a$. The ratchet consists of modifying at constant velocity the parameters $V_{1}$ and $V_{2}$ along the path depicted in the same figure (center). This example is a modification of the one presented in ref. 11. Now $\phi_{0}$ does not vanish and the above theory gives us the efficiency in the limit $T \rightarrow \infty$ and $F \rightarrow 0$. For instance, for $V=5$ and $a=0.2$, we obtain $\phi_{0}=0.825$, 
$\bar{\mu}=0.094$ and $b=3.74$. The efficiency given by eq. (11) is plotted in fig. 3 (right) and it is compared with the numerical integration of the Smoluchowski equation for different values of $T$. Notice the differences with the irreversible ratchet. Here the efficiency is an increasing function of $T$. The maximum efficiency, for the parameters corresponding to fig. 3, is $26 \%$ and is almost reached for $T=40$. The efficiency of this ratchet can be arbitrarily close to $100 \%$ if one increases $V$. The reason is that the average mobility decreases exponentially with $V$, but the coefficient $b$ and the integrated flow $\phi_{0}$ remain finite. For instance, for $V=20$ and $a=0.4, \phi_{0}=0.999988$, $b=6.89$ and $\bar{\mu}<10^{-7}$, yielding a maximum efficiency of $99.85 \%$.

To summarize, we have calculated the efficiency of a randomly flashing ratchet with an asymmetric sawtooth potential. In order to find more efficient Brownian motors, we have also calculated the efficiency of deterministically driven ratchets, finding that the efficiency of reversible ratchets is much higher than the efficiency of irreversible ratchets. It is remarkable that the class of reversible ratchets involves potentials depending on two or more parameters [11 and they differ from the models considered to date in the literature. Here we have shown that this new and non trivial class of ratchets is a real breakthrough regarding efficiency.

This work has been financially supported by Dirección General de Investigación Cientifica y Técnica (DGICYT) (Spain) Project No. PB94-0265. J.M.B. and F.J.C. acknowledge financial support from the program Becas de Colaboración of the Ministerio de Educación (Spain).

\section{References}

[1] Magnasco M.O., Phys. Rev. Lett., 71 (1993) 1477.

[2] Prost J., Chawin J.-F., Peliti L., and Adjari A., Phys. Rev. Lett., 72 (1994) 2652.

[3] Astumian R.D. and Bier M., Phys. Rev. Lett., 72 (1994) 1766.

[4] Doering Ch.R., Horsthemke W., and Riordan J., Phys. Rev. Lett., 72 (1994) 2984.

[5] Bartussek R., Hänggi P., And Kissner J.G., Europhys. Lett., 28 (1994) 459.

[6] Jülicher F., Ajdari A., And Prost J., Rev. Mod. Phys., 69 (1997) 1269.

[7] Feynman R.P., Leighton R.B., and Sands M., The Feynman Lectures on Physics, vol. 1 (Addison Wesley, Reading, Massachusetts), 1963 secs. 46.1-46.9.

[8] Parrondo J.M.R. and Español P., Am. J. Phys., 64 (1996) 1125.

[9] Seкimoto K., J. Phys. Soc. Jap., 66 (1997) 1234.

[10] Sokolov I.M. and Blumen A., J. Phys. A, 30 (1997) 3021.

[11] Parrondo J.M.R., Reversible ratchets as Brownian particles moving in an adiabatically changing periodic potential. Phys. Rev. E, in press. 